\title{
Symmetric Tensor Rank and Scheme Rank: An Upper Bound in terms of Secant Varieties
}

\author{
E. Ballico \\ Department of Mathematics, University of Trento, Povo, 38123 Trento, Italy \\ Correspondence should be addressed to E. Ballico; ballico@science.unitn.it
}

Received 3 June 2013; Accepted 9 August 2013

Academic Editor: Anna Fino

Copyright (C) 2013 E. Ballico. This is an open access article distributed under the Creative Commons Attribution License, which permits unrestricted use, distribution, and reproduction in any medium, provided the original work is properly cited.

Let $X \subset \mathbb{P}^{r}$ be an integral and nondegenerate variety. Let $c$ be the minimal integer such that $\mathbb{P}^{r}$ is the $c$-secant variety of $X$, that is, the minimal integer $c$ such that for a general $O \in \mathbb{P}^{r}$ there is $S \subset X$ with $\#(S)=c$ and $O \in\langle S\rangle$, where \langle\rangle is the linear span. Here we prove that for every $P \in \mathbb{P}^{r}$ there is a zero-dimensional scheme $Z \subset X$ such that $P \in\langle Z\rangle$ and $\operatorname{deg}(Z) \leq 2 c$; we may take $Z$ as union of points and tangent vectors of $X_{\text {reg }}$.

\section{Introduction}

There is a huge literature on the rank of tensors, on the symmetric tensor rank of symmetric tensors, and on the Waring decomposition of multivariate polynomials [1-14]. Most of the papers are over $\mathbb{C}$ (or over an algebraically closed field), but real tensors and real polynomials are also quite studied $[6,15]$. In this paper we work over an algebraically closed field $\mathbb{K}$ such that $\operatorname{char}(\mathbb{K})=0$ (e.g., $\mathbb{C}$ ), but for homogeneous polynomials we also work over $\mathbb{R}$ (see Corollary 3). Let $X \subset \mathbb{P}^{r}$ be an integral and nondegenerate variety. Fix $P \in \mathbb{P}^{r}$. A tangent vector of $X$ or a tangent vector of $X_{\text {reg }}$ or a smooth tangent vector of $X$ is a zero-dimensional connected subscheme of $X$ whose support is a smooth point of $X$, that is, a point of $X_{\text {reg }}$, and with degree 2. Fix $O \in X_{\text {reg }}$ and let $m$ be the dimension of $X$ at $O$. The set of all smooth tangent vectors of $X$ with $O$ as its support is parametrized by a projective space of dimension $m-1$. If $\mathbb{K}=\mathbb{C}, X$ is defined over $\mathbb{R}$ and $O \in X_{\text {reg }}(\mathbb{R})$, a smooth tangent vector $Z \subset X$ with $Z_{\text {red }}=\{O\}$ is said to be real if it is defined over $\mathbb{R}$. A zero-dimensional scheme $Z \subset X$ is said to be curvilinear if for each connected component $W$ of $Z$ either $W$ is a point of $X$ or there is $W_{\text {red }} \in X_{\text {reg }}$ and $W$ is contained in a smooth curve contained in an open neighborhood of $W_{\text {red }}$ in $X$. A zero-dimensional scheme $Z \subset X$ is said to be smoothable if it is a flat limit of a flat family of finite subsets of $X$ (a curvilinear scheme is smoothable). Fix $P \in \mathbb{P}^{r}$. The $X$-rank $r_{X}(P)$ of $P$ is the minimal cardinality of a finite set $S \subset X$ such that
$P \in\langle S\rangle$, where \langle\rangle denote the linear span. The scheme $X$-rank (or $X$-cactus rank) $z_{X}(P)$ of $P$ is the minimal degree of a zerodimensional scheme $Z \subset X$ such that $P \in\langle Z\rangle[16$, Definition 5.1, page 135, Definition 5.66, page 198, 31, 12, 10, 11, 17, 18, 8, 9]. If we impose that $Z$ is smoothable (curvilinear, resp.), then we get the smoothable $X$-rank $z_{X}^{\prime}(P)$ (curvilinear $X$-rank $z_{X}^{\prime \prime}(P)$, resp.) of $[17,18]$ for wonderful uses of the scheme $X$-rank. Let $w_{X}(P)$ be the minimal degree of a zero-dimensional scheme $Z \subset X$ such that $P \in\langle Z\rangle$ and each connected component of $Z$ is either a point of $X$ or a smooth tangent vector of $X$ (any such $Z$ is curvilinear). We have

$$
z_{X}(P) \leq z_{X}^{\prime}(P) \leq z_{X}^{\prime \prime}(P) \leq w_{X}(P) .
$$

Hence to get an upper bound for the integer $z_{X}(P)$, it is sufficient to find an upper bound for the integer $w_{X}(P)$. We first state our upper bound in the case of the Veronese varieties (this case corresponds to the decomposition of homogeneous polynomials as a sum of powers of linear forms).

For all positive integers $m$ and $d$ let $v_{d}: \mathbb{P}^{m} \rightarrow \mathbb{P}^{r}, r:=$ $\left(\begin{array}{c}m+d \\ m\end{array}\right)-1$, denote the order $d$ Veronese embedding of $\mathbb{P}^{m}$, that is, the embedding of $\mathbb{P}^{m}$ given by the $\mathbb{K}$-vector space of all degree $d$ homogeneous polynomials in $m+1$ variables.

Theorem 1. Fix integers $m \geq 2$ and $d \geq 3$. If $m \leq 4$, then assume $d \geq 5$. Let $X_{m, d} \subset \mathbb{P}^{r}, r:=\left(\begin{array}{c}m+d \\ m\end{array}\right)-1$, be the order $d$ Veronese embedding of $\mathbb{P}^{m}$. Set $c:=\left\lceil\left(\begin{array}{c}m+d \\ m\end{array}\right) /(m+1)\right\rceil$. Let 
$\Omega \subset X_{m, d}$ be any nonempty open subset of $X_{m, d}$. Then there is a disjoint union $Z \subset \Omega$ of c tangent vectors such that $P \in\langle Z\rangle$.

Corollary 2. In the setup of Theorem 1 , one has $w_{X_{m, d}}(P) \leq$ $2\left\lceil\left(\begin{array}{c}m+d \\ m\end{array}\right) /(m+1)\right\rceil$ for all $P \in \mathbb{P}^{r}$.

Corollary 3. Let $X_{m, d}(\mathbb{R}) \subset \mathbb{P}^{r}(\mathbb{R}), r:=\left(\begin{array}{c}m+d \\ m\end{array}\right)-1$, be the order $d$ Veronese embedding of $\mathbb{P}^{m}(\mathbb{R})$. Fix $P \in \mathbb{P}^{r}(\mathbb{R})$ and a nonempty open subset $U \subseteq \mathbb{P}^{m}(\mathbb{R})$ for the euclidean topology. Then there is $S \subset U$ and for each $Q \in S$ a real tangent vector $v_{Q}$ of $\mathbb{P}^{m}(\mathbb{R})$ such that $\#(S) \leq\left\lceil\left(\begin{array}{c}m+d \\ m\end{array}\right) /(m+1)\right\rceil$ and $P \in\left\langle\cup_{\mathrm{Q} \in S} v_{d}\left(v_{\mathrm{Q}}\right)\right\rangle$.

Theorem 1 is just a particular case of a general bound on $w_{X}(P)$ (see Theorem 4 ). We want to point out two features of these results.

(i) The use of an arbitrary nonempty open subset $\Omega$ $\left(U\right.$, resp.) of $X_{m, d}\left(\mathbb{P}^{m}(\mathbb{R})\right.$, resp.). This is not just to get a formally stronger statement. In many cases, the inductive proofs require the existence of sets (or schemes) bounding $r_{X}(P)$ or $z_{X}(P)$ and with supports away from some bad varieties $[10,11,14,19]$. For instance, in [19] Jelisiejew takes as $\Omega$ the image by the Veronese embedding $\nu_{d}$ of the complement of finitely many hyperplanes; he calls it the "open rank."

(ii) We use very particular curvilinear schemes, just disjoint unions of tangent vectors. One should find algorithms to find the support and the direction of tangent vectors needed to compute a good upper bound for the integer $w_{X}(P)$.

Let $X \subset \mathbb{P}^{r}$ be an integral and nondegenerate variety. Set $m:=\operatorname{dim}(X)$. For each integer $b>0$ the $b$-secant variety $\sigma_{b}(X) \subseteq \mathbb{P}^{r}$ of $X$ is the closure in $\mathbb{P}^{r}$ of the union of all linear spaces $\langle S\rangle$, where $S \subset X$ is a subset with cardinality $b$. The set $\sigma_{b}(X)$ is an integral variety of dimension at $\operatorname{most} \min \{r,(m+$ $1) b-1\}$. In many important cases, the integer $\operatorname{dim}\left(\sigma_{b}(X)\right)$ is known and either $\operatorname{dim}\left(\sigma_{b}(X)\right)=\min \{r,(m+1) b-1\}$ or $\operatorname{dim}\left(\sigma_{b}(X)\right)-\min \{r,(m+1) b-1\}$ is very small $[20,21]$. Hence it is usually easy to find an integer $c$ with $c-\lceil(r+1) /(m+1)\rceil$ small such that $\sigma_{c}(X)=\mathbb{P}^{r}$.

Theorem 4. Let $X \subset \mathbb{P}^{r}$ be an integral and nondegenerate variety. Let $c$ be the first positive integer such that $\sigma_{c}(X)=\mathbb{P}^{r}$. Fix any nonempty open subset $U$ of $X$ and any $P \in \mathbb{P}^{r}$. Then one has the following.

(a) There is a disjoint union $Z \subset U$ of $c$ smooth tangent vectors such that $P \in\langle Z\rangle$;

(b) $w_{X}(P) \leq 2 c$.

Remark 5. Take $X$ and $c$ as in Theorem 4. We have $c=r_{X}(O)$ with $O$ a general element of $\mathbb{P}^{r}$. Hence the scheme $X$-rank of the worst point of $\mathbb{P}^{r}$ is at most twice the rank of almost all points of $\mathbb{P}^{r}$.

\section{The Proofs}

Proof of Theorem 4. Fix a general $S \subset U \cap X_{\text {reg }}$ such that $\#(S)=c$. For each $O \in X_{\text {reg }}$ let $T_{O} X \subset \mathbb{P}^{r}$ denote the Zariski tangent space of $X$ at $O$. Since $\sigma_{c}(X)=\mathbb{P}^{r}$, Terracini's lemma gives $\mathbb{P}^{r}=\left\langle\cup_{O \in S} T_{O} X\right\rangle$ [20, Corollary 1.11]. Hence for each $O \in S$, there is $P_{O} \in T_{O} X$ such that $P \in\left\langle\cup_{O \in S} P_{O}\right\rangle$. Fix $O \in S$. If $P_{O}=O$, then let $v_{O}$ be any tangent vector of $X$ at $O$. Now assume that $P_{O} \neq O$. Since the line $L:=\left\langle\left\{P_{O}, O\right\}\right\rangle$ is contained in $T_{O} X$, the scheme $L \cap X$ contains the tangent vector $v_{O}$ of $L$ at $O$. Set $Z:=\cup_{O \in S} v_{O}$. Since $P_{O} \in\left\langle v_{O}\right\rangle$ for all $O \in S$, we have $P \in\langle Z\rangle$.

Proof of Theorem 1. Since either $m \geq 5$ and $d \geq 3$ or $d \geq$ 5 , a theorem of Alexander and Hirschowitz says that $c$ is the first positive integer $t$ such that $\sigma_{t}\left(X_{m, d}\right)=\mathbb{P}^{r}$ [22-25]. Apply Theorem 4.

Proof of Corollary 2. This is a consequence of Theorem 1.

Proof of Corollary 3. For any subset $A$ of $\mathbb{P}^{r}(\mathbb{R})$ we write $\langle A\rangle$ for its linear span over $\mathbb{C}$ and $\langle A\rangle_{\mathbb{R}}$ for its linear span over $\mathbb{R}$. For each $Q \in X_{m, d}(\mathbb{R})$, we write $T_{Q} X_{m, d}(\mathbb{C})$ for the Zariski tangent space over $\mathbb{C}$ and write $T_{Q} X_{m, d}(\mathbb{R})$ for the real tangent space (hence both projective spaces have dimension $m$, the first one over $\mathbb{C}$, the second one over $\mathbb{R})$. We have $T_{\mathrm{Q}} X_{m, d}(\mathbb{C}) \cap \mathbb{P}^{r}(\mathbb{R})=T_{\mathrm{Q}} X_{m, d}(\mathbb{R})$. The set $v_{d}(U)$ is Zariski dense in $X_{m, d}(\mathbb{C})$. Hence Terracini's lemma gives the existence of $S \subset U$ such that $\#(S)=a$ and $\left\langle\cup_{\mathrm{Q} \in v_{d}(S)} T_{\mathrm{Q}} X_{m, d}(\mathbb{C})\right\rangle=\mathbb{P}^{r}(\mathbb{C})$. Since $\nu_{d}(S) \subset X_{m, d}(\mathbb{R})$ and $P \in \mathbb{P}^{r}(\mathbb{R})$, we get $P \in\left\langle\cup_{\mathrm{O} \in v_{d}(S)} T_{O} X_{m, d}(\mathbb{R})\right\rangle_{\mathbb{R}}$. Hence for each $O \in v_{d}(S)$ there is $P_{O} \in T_{O} X_{m, d}(\mathbb{R})$ such that $P \in$ $\left\langle\cup_{\mathrm{O} \in v_{d}(S)} P_{\mathrm{O}}\right\rangle_{\mathbb{R}}$. Continue as in the proof of Theorem 4 .

\section{Conflict of Interests}

The author declares that there is no conflict of interests regarding the publication of this paper.

\section{Acknowledgment}

The author was partially supported by MIUR and GNSAGA of INdAM (Italy).

\section{References}

[1] J. Brachat, P. Comon, B. Mourrain, and E. Tsigaridas, "Symmetric tensor decomposition," Linear Algebra and its Applications, vol. 433, no. 11-12, pp. 1851-1872, 2010.

[2] J. Buczyński and J. M. Landsberg, "Ranks of tensors and a generalization of secant varieties," Linear Algebra and its Applications, vol. 438, no. 2, pp. 668-689, 2013.

[3] P. Comon, G. Golub, L.-H. Lim, and B. Mourrain, "Symmetric tensors and symmetric tensor rank," SIAM Journal on Matrix Analysis and Applications, vol. 30, no. 3, pp. 1254-1279, 2008.

[4] J. M. Landsberg, Tensors: Geometry and Applications, vol. 128 of Graduate Studies in Mathematics, American Mathematical Society, Providence, RI, USA, 2012. 
[5] J. M. Landsberg and Z. Teitler, "On the ranks and border ranks of symmetric tensors," Foundations of Computational Mathematics, vol. 10, no. 3, pp. 339-366, 2010.

[6] V. de Silva and L.-H. Lim, "Tensor rank and the ill-posedness of the best low-rank approximation problem," SIAM Journal on Matrix Analysis and Applications, vol. 30, no. 3, pp. 1084-1127, 2008.

[7] J. Kleppe, Representing a homogeneous polynomial as a sum of powers of linear forms [Thesis for the degree of Candidatum Scientiarum], Department of Mathematics, University of Oslo, 1999, http://folk.uio.no/johannkl/kleppe-master.pdf.

[8] G. Ottaviani, "An invariant regarding Waring's problem for cubic polynomials," Nagoya Mathematical Journal, vol. 193, pp. 95-110, 2009.

[9] E. Ventura, "A note on the Waring ranks of reducible cubicforms," http://arxiv.org/abs/1305.5394.

[10] A. Białynicki-Birula and A. Schinzel, "Representations of multivariate polynomials by sums of univariate polynomials in linear forms," Colloquium Mathematicum, vol. 112, no. 2, pp. 201-233, 2008.

[11] A. Białynicki-Birula and A. Schinzel, "Corrigendum to 'Representatons of multivariate polynomials by sums of univariate polynomials in linear forms"', Colloquium Mathematicum, vol. 125, no. 1, article 139, 2011.

[12] E. Ballico, "An upper bound for the symmetric tensor rank of a low degree polynomial in a large number of variables," ISRNGeometry, vol. 2013, Article ID 715907, 2 pages, 2013.

[13] E. Ballico, "An upper bound for the tensor rank of an n-tensor," submitted.

[14] E. Ballico, "An upper bound for the tensor rank," ISRN Geometry, vol. 2013, Article ID 241835, 3 pages, 2013.

[15] P. Comon and G. Ottaviani, "On the typical rank of real binary forms," Linear and Multilinear Algebra, vol. 60, no. 6, pp. 657667, 2012.

[16] A. Iarrobino and V. Kanev, Power Sums, Gorenstein Algebras, and Determinantal Loci, vol. 1721 of Lecture Notes in Mathematics, Springer, Berlin, Germany, 1999, Appendix C by Iarrobino and Steven L. Kleiman.

[17] W. Buczyńska and J. Buczyński, "Secant varieties to high degree veronese reembeddings, catalecticant matrices and smoothable Gorenstein schemes," http://arxiv.org/abs/1012.3563 .

[18] J. Buczyński, A. Ginensky, and J. M. Landsberg, "Determinantal equations for secant varieties and the Eisenbud-Koh-Stillman conjecture," Journal of the London Mathematical Society, vol. 88, no. 2, pp. 1-24, 2013.

[19] J. Jelisiejew, "An upper bound for the Waring rank of a form," http://arxiv.org/abs/1305.6957.

[20] B. Ådlandsvik, "Joins and higher secant varieties," Mathematica Scandinavica, vol. 61, no. 2, pp. 213-222, 1987.

[21] B. Ådlandsvik, "Varieties with an extremal number of degenerate higher secant varieties," Journal für die Reine und Angewandte Mathematik, vol. 392, pp. 16-26, 1988.

[22] J. Alexander and A. Hirschowitz, "La méthode d'Horace éclatée: application à l'interpolation en degré quatre," Inventiones Mathematicae, vol. 107, no. 3, pp. 585-602, 1992.

[23] J. Alexander and A. Hirschowitz, "Polynomial interpolation in several variables," Journal of Algebraic Geometry, vol. 4, no. 2, pp. 201-222, 1995.

[24] K. A. Chandler, "A brief proof of a maximal rank theorem for generic double points in projective space," Transactions of the
American Mathematical Society, vol. 353, no. 5, pp. 1907-1920, 2001.

[25] M. C. Brambilla and G. Ottaviani, "On the AlexanderHirschowitz theorem," Journal of Pure and Applied Algebra, vol. 212, no. 5, pp. 1229-1251, 2008.

[26] A. Bernardi, J. Brachat, and B. Mourrain, "A comparison of different notions of ranks of symmetric tensors," http://arxiv.org/abs/1210.8169.

[27] A. Bernardi, P. Macias, and K. Ranestad, "Computing the cactus rank of a general form," http://arxiv.org/abs/1211.7306.

[28] W. Buczyńska and J. Buczyński, "On the difference between the border rank and the smoothable rank of a polynomial," http://arxiv.org/abs/1305.1726.

[29] E. Ballico and A. Bernardi, "Curvilinear schemes and maximum rank of forms," http://arxiv.org/abs/1210.8171. 


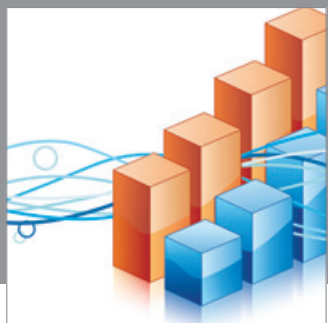

Advances in

Operations Research

mansans

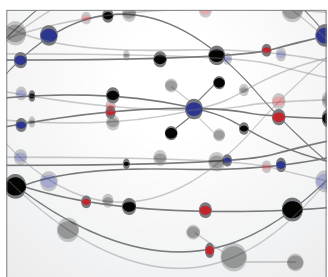

The Scientific World Journal
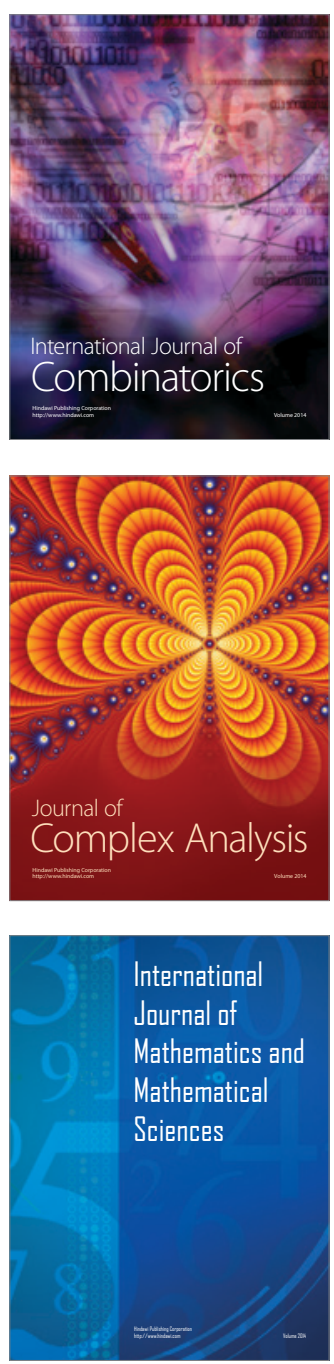
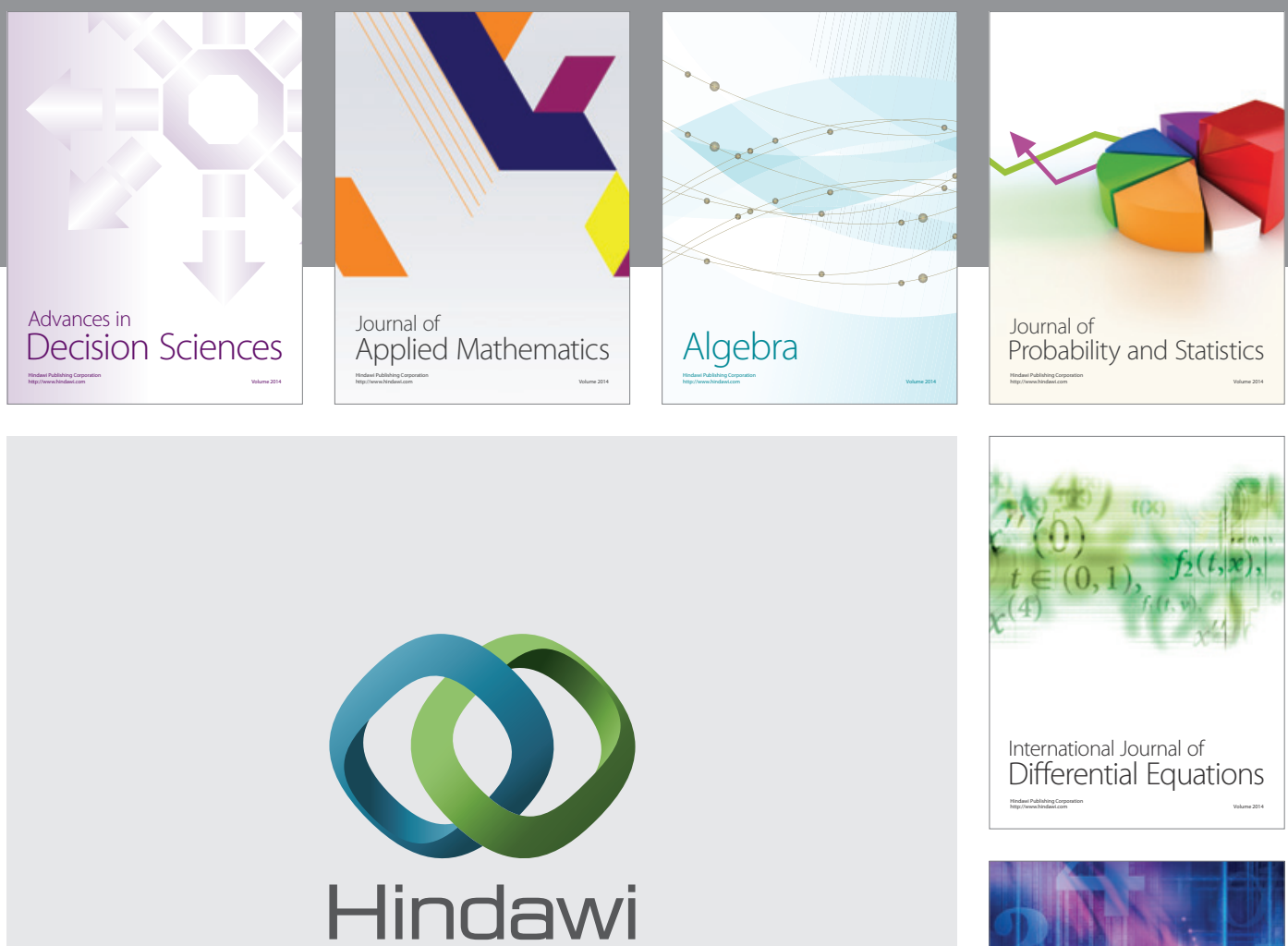

Submit your manuscripts at http://www.hindawi.com
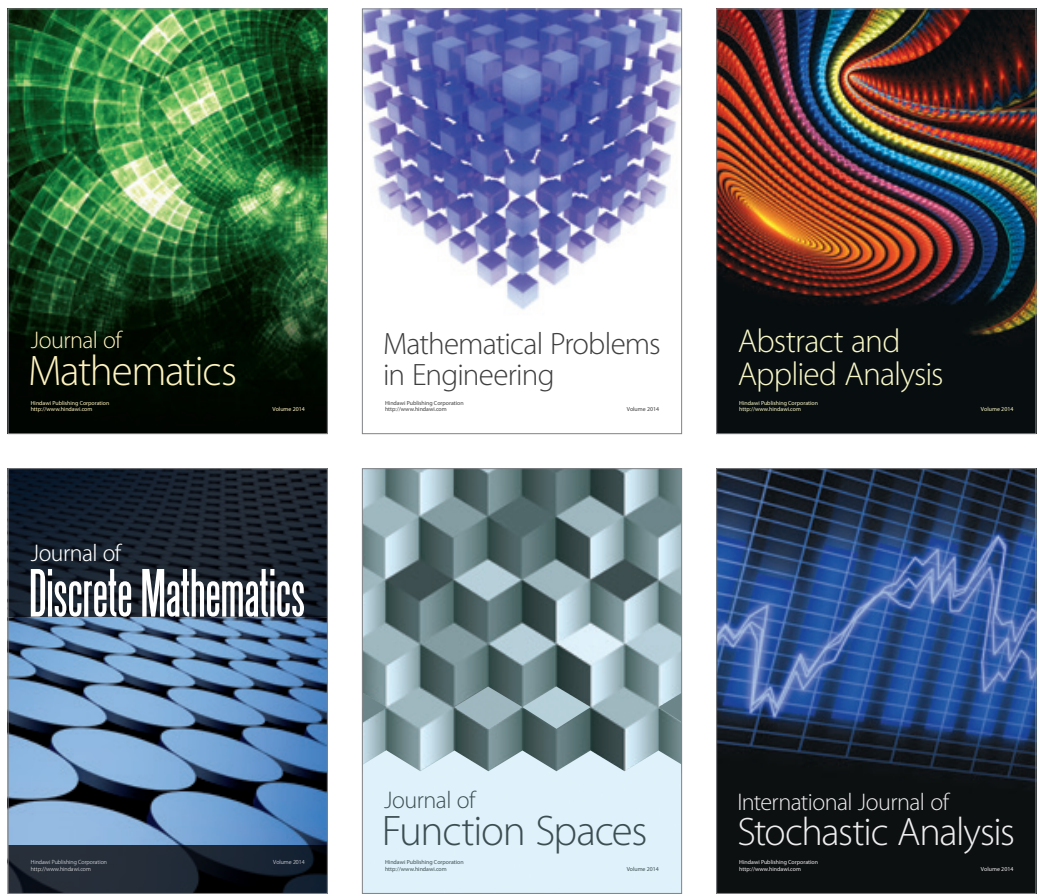

Journal of

Function Spaces

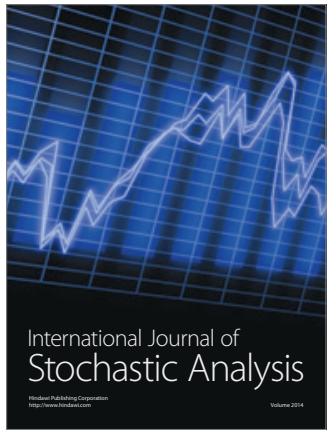

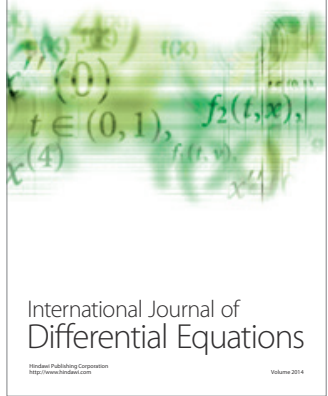
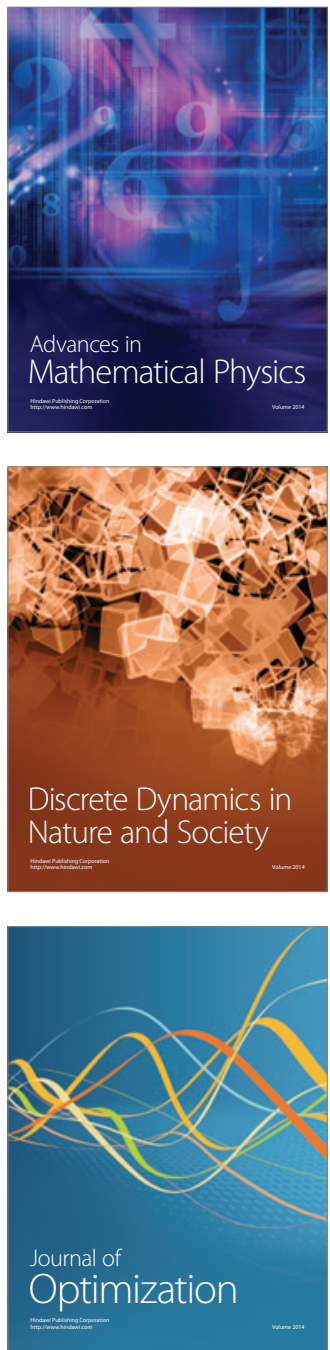